$393)$, dementia $(\mathrm{n}=9)$ and other $(\mathrm{n}=311)$. The lowest mortality was seen in patients with HNC (30 day mortality $=5.2 \%, 1$ year mortality $=32.6 \%)$. In comparison, 30 day mortality in all other groups was significantly higher $(8.47 \%$ in neurological diseases, $15.86 \%$ in dysphagic stroke, $33.3 \%$ in dementia and $11.25 \%$ in 'other' indication, $\mathrm{p}<0.01)$. Mortality was also significantly higher at one year $(\mathrm{p}<0.01)$. There was no significant difference in mortality when comparing radiologically inserted and percutaneous endoscopic gastrostomies. Higher mortality rates were seen in patients aged 60 years or above at 30 days (OR $2.439(1.666-3.731) \mathrm{p}<0.0001)$ and also at 1 year (OR $3.140(0.268-0.600) \mathrm{p}<0.0001)$. Albumin less than $30 \mathrm{~g} / \mathrm{L}$ was also associated with significantly higher 30 day (OR $4.486(3.067-6.561) \mathrm{p}<0.0001)$ and 1 year mortality outcomes (OR 2.319 (1.830 to 2.939) p < 0.0001). In accordance with recent published data, our findings would support an elevated CRP ( $>5 \mathrm{mg} / \mathrm{L}$ ) being a factor associated with 30 day mortality (OR 8.930 (1.199 to 66.51) p = 0.006).

Conclusion Referral indication for gastrostomy significantly impacts 30 day and 1 year mortality outcomes, with lowest rates demonstrated in patients with HNC. Identification of factors associated with mortality as seen in this study could help improve patient selection and be of relevance in the decision making process for gastrostomy.

Disclosure of Interest None Declared.

\section{OC-024 PREDICTING 30-DAY MORTALITY FOLLOWING PEG INSERTION: EXTERNAL VALIDATION OF A PREVIOUS SCORING SYSTEM AND ANALYSIS FOR ADDITIONAL PREDICTIVE FACTORS}

CS MacLeod, R McKay, D Barber, AW Mckinlay, JS Leeds*. Gastroenterology, Aberdeen Royal Infirmary, Aberdeen, UK

\subsection{6/gutjnl-2014-307263.24}

Introduction Percutaneous endoscopic gastrostomy (PEG) insertion is a well-established technique for providing long-term enteral nutrition. However concerns have been raised regarding the high 30-day mortality associated with PEG, and the related ethical implications of patient selection. Accordingly, a previous predictive tool was developed using age and serum albumin level but was created on a relatively small cohort. External validation of the score was performed in the same region but has not been outside of this area. This study aimed to externally validate this previous scoring system and also try to identify any further predictors of 30-day mortality in a larger cohort.

Methods Retrospective review of all gastroscopy reports documenting PEG insertions between January 2001 and January 2012 in our centre was undertaken. Hospital electronic systems were used to determine patient demographics, laboratory results and outcome at 30 days. In patients with newly inserted PEG tubes, the scoring system was applied and assessed using receiver operating curve analysis to determine the discriminative capacity. Furthermore, univariate and multivariate binary logistic regression analyses were performed using the current database to identify additional predictors of 30-day mortality.

Results The PEG database included 1373 patients, of which 808 were new PEG insertions and suitable for analysis. For each increasing gradation of the scoring system, mortality rose with $4 \%$ of those scoring zero dying compared to $50 \%$ scoring three. An area under the ROC curve of 0.686 (95\% confidence interval $0.635-0.737$ ) indicated reasonable discriminative capacity. Multivariate analysis demonstrated that age $\geq 60$ years
$(\mathrm{OR}=2.097[\mathrm{p}=0.016])$, serum albumin levels of $25-34 \mathrm{~g} / \mathrm{l}$ $(\mathrm{OR}=2.447[\mathrm{p}=0.001])$ or $<25 \mathrm{~g} / \mathrm{l}(\mathrm{OR}=6.769[\mathrm{p}<$ $0.001])$, C-Reactive Protein $\geq 10 \mathrm{mg} / \mathrm{l}(\mathrm{OR}=2.713[\mathrm{p}=$ $0.009])$ and lymphocyte count of $<1.5 \times 10^{9} / 1(\mathrm{OR}=2.016[\mathrm{p}$ $=0.004]$ ) increased the odds of 30 -day mortality, whilst inpatient PEG placement decreased the risk of death $(\mathrm{OR}=0.529$ $[\mathrm{p}=0.005])$.

Conclusion The previous scoring system demonstrated reasonable predictive proficiency but the area under the ROC curves were not $>0.8$. The recognition of further predictors of 30-day mortality allows for remodelling of the score which may improve the accuracy. However, future prospective, multicentre studies with defined outcomes are necessary to improve data collection. Additionaly, more information is needed about cause of 30-day mortality and importantly quality of life following PEG insertion.

Disclosure of Interest None Declared.

\section{OC-025 SURVIVAL OF PATIENTS WITH PALLIATIVE INOPERABLE GASTROINTESTINAL OBSTRUCTION DUE TO MALIGNANCY TREATED WITH HOME PARENTERAL NUTRITION}

${ }^{1} \mathrm{M}$ Naghibi*, ${ }^{1} \mathrm{M}$ Stroud, ${ }^{1} \mathrm{TR}$ Smith, ${ }^{2} \mathrm{M}$ Elia. ${ }^{1}$ Gastroenterology, University Hospital Southampton, Southampton, UK; ${ }^{2}$ Institute of Human Nutrition, University of Southampton, Southampton, UK

\subsection{6/gutjnl-2014-307263.25}

Introduction There is controversy about the indications for home parenteral nutrition (HPN) during the palliative phase of malignancy causing inoperable gastrointestinal obstruction (IBO). This is partly due to uncertainty about the survival of patients. This study aimed to establish the survival characteristics of these patients in order to inform decisions about the use of HPN.

Methods A systematic review with meta-analyses were carried out in accordance with the Cochrane protocol for adult patients ( $>18$ years) with a confirmed diagnosis of malignancy causing IBO (in at least $80 \%$ of the patients) being treated with palliative HPN. A literature search was carried out in April 2013 using Medline, EMBASE, CINALH and Web of knowledge. Whenever possible, individual patient data were extracted to allow metaanalyses.

Results 11 studies involving 420 patients, met the inclusion criteria. 3 studies reported individual patient data, 4 studies represented this using Kaplan Meier, one study using scatter plot and 3 studies only reported averages for survival length. The extraction procedure which gathered individual information on

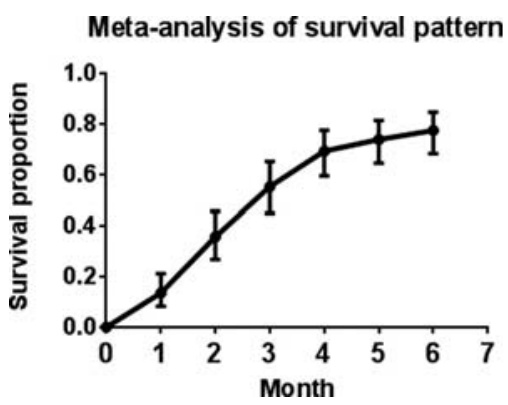

Abstract OC-025 Figure 1 Random effects meta-analyses of survival at monthly intervals up to 6 months ( $n=220$ patients; 8 studies). The bars represent the $95 \%$ confidence intervals 
220 patients from 8 studies, allowed a random effects model meta-analysis to be carried out. The mean age of patients within individual studies ranged between 45-61 years. 64\% of patients were female. The underlying primary malignancy was gastrointestinal (53\%), gynaecological (25\%) (accounting for the female predominance), and others (22\%). Four studies reported use of palliative chemoradiotherapy with $39-100 \%$ of the patients under going at least one cycle of treatment. Figure 1 shows the survival at monthly intervals for six months. The overall median survival is 83 days. $24 \%$ were still alive at 6 months but only about $2 \%$ at year.

Conclusion This is first systematic review showing the survival in patients with malignant inoperable bowel obstruction receiving HPN during the palliative phase of care. We show, using the largest published cohort to date, that the median survival is only 83 days. The described variability in survival length between studies and between patients can be substantial. This information can help inform clinician decisions about the use of HPN in such patients.

Disclosure of Interest None Declared.

\section{OC-026 DIAGNOSTIC YIELDS OF SEHCAT SCANNING AND GLUCOSE HYDROGEN BREATH TESTING IN PELVIC RADIATION DISEASE}

R Stacey*, J Turner, J Green. Department of Gastroenterology, University Hospital Llandough, Cardiff, UK

\subsection{6/gutjnl-2014-307263.26}

Introduction Late Gastrointestinal side effects following radiotherapy are common but under reported by patients and poorly recognised by clinicians ${ }^{-1}$ Pelvic radiation disease (PRD) can present up to 30 years after radiotherapy treatment with a range of symptoms, including rectal bleeding, abdominal pain and diarrhoea. Many of these patients experience troublesome symptoms of diarrhoea secondary to Bile acid Malabsorption (BAM) and small intestinal bacterial overgrowth (SIBO).

In our centre we have access to 23-seleno-25-homo-taurocholic acid (SeHCAT) nuclear medicine scanning and glucose hydrogen breath testing for the investigation of BAM and SIBO associated with PRD. The majority of data on patients with pelvic radiation disease has been published by one specialist centre (The Royal Marsden Hospital). We audited the diagnostic yields for investigation of small intestinal manifestations of PRD.

Methods We identified 77 patients referred to gastroenterology clinic at University Hospital Llandough, Cardiff with suspected pelvic radiation disease via a departmental database. All patients were investigated via the Royal Marsden algorithm and BSG guidance. We then identified all patients with diarrhoeal symptoms and audited the results of any SeHCAT and glucose hydrogen breath tests and their diagnostic yields.

Results 26 patients (36\%) had diarrhoeal symptoms. Within this group, 24 patients underwent SeHCAT scanning, with $45 \%$ having positive results. 19 were referred for glucose hydrogen breath tests of which $53 \%$ had positive results. $17 \%$ had both positive SeHCAT and Glucose hydrogen breath tests. Not all patients received both tests due to various reasons (clinical decision, patient choice and non attendance).

Conclusion PRD is common but under investigated. BSG guidance on its management exists ${ }^{[1]}$ and an algorithmic approach has been shown to be beneficial. ${ }^{2}$ Diarrhoea occurs frequently in
PRD, and we have found that SeHCAT scanning and Glucose Hydrogen breath tests in these patients have a high diagnostic yield for BAM and SIBO respectively. SIBO and BAM can exist concurrently in this group, and our data supports a thorough and systematic algorithmic approach. Clinicians should have a low threshold for requesting these tests in patients with suspected PRD.

\section{REFERENCES}

1 Andreyev HJN, Davidson SE, Gillespie C, Allum WH, Swarbrick E. British Society of Gastroenterology, et al. Practice guidance on the management of acute and chronic gastrointestinal problems arising as a result of treatment for cancer. Gut 2012:179-92

2 Andreyev HJN, Benton BE, Lalji A, Norton PC, Kabir M, Gage PH, et al. ArticlesAlgorithm-based management of patients with gastrointestinal symptoms in patients after pelvic radiation treatment (ORBIT): a randomised controlled trial. Lancet. Elsevier Ltd; 2013 Sep 20:1-9

Disclosure of Interest None Declared.

\section{OC-027 FGF19 LEVELS IN SUBJECTS WITH PRIMARY BILE ACID DIARRHOEA AND ELEVATED TRIGLYCERIDES}

${ }^{1} \mathrm{~J}$ Nolan, ${ }^{2} \mathrm{~S}$ Pattni, ${ }^{1} \mathrm{JR}$ Walters, IM Johnston*. 'Imperial College London, London, UK; ${ }^{2}$ University of Leicester, Leicester, UK

\subsection{6/gutjnl-2014-307263.27}

Introduction There is evidence that primary bile acid diarrhoea (PBAD) is caused by disordered bile acid homeostasis. Most patients with severe PBAD have low fasting serum FGF19 which fails to rise above $300 \mathrm{pg} / \mathrm{ml}$ postprandially. Different patterns of postprandial FGF19 response have been demonstrated, with some resembling those of healthy individuals. Others have shown that serum triglyceride levels reflect expression of the luminal bile acid transporter ASBT. It is hypothesised that a subset of individuals with hypertriglyceridaemia have different fasting FGF19 levels and postprandial FGF19 response.

Methods Study 1: 162 patients with chronic diarrhoea were recruited prospectively. All patients underwent routine testing to exclude other causes of diarrhoea and had SeHCAT tests. Patients were classified as having PBAD, or unexplained chronic diarrhoea (CD). Other diagnoses were excluded. Fasting blood samples were taken and processed for triglycerides and FGF19. Subjects with either diagnosis were also analysed within 2 subgroups according to triglyceride level (cut off $2.30 \mathrm{mmol} / \mathrm{l})$. Study 2: 18 subjects took part in a study to examine FGF19 levels over the course of $6 \mathrm{~h}$. After an overnight fast, blood was sampled every $90 \mathrm{~min}$ for $6 \mathrm{~h}$. Meals were provided at 9 am and 12 noon. Serum FGF19 was quantified by ELISA using a commercially available kit. Triglycerides were quantified by standard colorimetric technique. Mann-Whitney and Spearman rank correlation tests were used in analyses.

Results Study 1: Overall subjects with elevated triglycerides $(\mathrm{n}=18)$ have significantly lower SeHCAT retention (median 7.95 vs. $19.5 \% \mathrm{p}=0.01)$. Subjects with severe BAD with elevated triglycerides had higher fasting FGF19 levels (241 vs $101 \mathrm{pg} / \mathrm{l} \mathrm{p}=0.02$ ). Study 2: There was no significant difference in fasting triglycerides between different phenotypes of FGF19 response (previously presented work). The percentage increase in FGF19 from fasting to $90 \mathrm{~min}$ after breakfast correlates with fasting serum triglyceride level $(\mathrm{R}=0.59, \mathrm{p}<0.005)$. 